\title{
Cardiovascular Disease in Cushing's Syndrome: Heart versus Vasculature
}

\author{
Monica De Leo Rosario Pivonello Renata S. Auriemma Alessia Cozzolino \\ Pasquale Vitale Chiara Simeoli Maria Cristina De Martino Gaetano Lombardi \\ Annamaria Colao \\ Department of Molecular and Clinical Endocrinology and Oncology, 'Federico II' University of Naples, Naples, Italy
}

\section{Key Words}

Cushing's syndrome $\cdot$ Cushing's disease $\cdot$ Cardiovascular disease $\cdot$ Cardiovascular risk - Cardiac abnormalities .

Vascular abnormalities - Cardiac hypertrophy ·

Hypertension • Atherosclerosis • Metabolic syndrome •

Coagulopathy

\begin{abstract}
Cushing's syndrome (CS) causes metabolic abnormalities that determine an increased cardiovascular risk not only during the active phase of the disease but also for a long time after cure. Cardiovascular complications, such as premature atherosclerosis, coronary artery disease, heart failure, and stroke, in patients with CS cause a mortality rate higher than that observed in a normal population. The increased cardiovascular risk is mainly due to metabolic complications, such as metabolic syndrome, but also to vascular and cardiac alterations such as atherosclerosis and cardiac structural and functional changes. In the clinical management of patients with CS the focus should be on identifying the global cardiovascular risk and the aim should be to control not only hypertension but also other correlated risk factors, such as obesity, glucose intolerance, insulin resistance, dyslipidemia, endothelial dysfunction and the prothrombotic state. Con-
\end{abstract}

sidering that remission from hypercortisolism is often difficult to achieve and that the cardiovascular risk can persist even during disease remission, care and control of all cardiovascular risk factors should be one of the primary goals during the follow-up of these patients.

Copyright $\odot 2010$ S. Karger AG, Basel

\section{Introduction}

Cushing's syndrome (CS) is characterized by a series of systemic complications, including visceral obesity, systemic arterial hypertension, impairment of glucose tolerance, dyslipidemia and thrombotic diathesis, which increase the cardiovascular risk [1]. Cardiovascular complications associated with CS include coronary artery disease, congestive heart failure and cardiac stroke, which significantly increase the mortality rate of CS patients compared with the normal population $[2,3]$. The remission or cure of CS usually reduces but does not completely eliminate these systemic complications. Therefore, a history of CS is associated with a persistent increased cardiovascular risk despite the normalization of cortisol secretion [4].

\section{KARGER}

Fax +41613061234 E-Mail karger@karger.ch www.karger.com
(C) 2010 S. Karger AG, Basel

0028-3835/10/0925-0050\$26.00/0

Accessible online at:

www.karger.com/nen
Rosario Pivonello

Department of Molecular and Clinical Endocrinology and Oncology

'Federico II' University of Naples, Via Sergio Pansini 5

IT-80131 Naples (Italy)

Tel. +39 081746 4737, Fax +39 349470 1356, E-Mail rpivone@ tin.it 


\section{The Metabolic Syndrome}

The increased cardiovascular risk of patients with CS is mainly due the metabolic syndrome [5]. The metabolic syndrome is a severe clinical condition associated with an increased cardiovascular risk, which affects approximately $20 \%$ of the general population and is characterized by visceral obesity, hypertension, insulin resistance and dyslipidemia, common features of patients with CS [6]. These metabolic abnormalities were found to be more severe in patients with pituitary-dependent CS or Cushing's disease (CD) who are characterized by abdominal obesity than in healthy subjects matched for body mass index who are characterized by general obesity, confirming the relevant impact of the visceral obesity on the development of the metabolic syndrome and the consequent increased cardiovascular risk [7]. Indeed, abdominal obesity, but not peripheral obesity, is associated with a decrease in peripheral insulin sensitivity and hepatic insulin extraction [6]. Insulin resistance is a prerequisite for the development of metabolic syndrome [6]. In patients with hypercortisolism, insulin resistance and abdominal obesity induce gluconeogenesis and promote a cascade of metabolic abnormalities that result in an overt metabolic syndrome [5]. In a transsectional study metabolic syndrome was found in patients who had been cured from $\mathrm{CD}$ for at least 5 years [8]. These patients maintained several clinical and biochemical abnormalities typical of the active phase of $\mathrm{CD}$, such as overweight or obesity in $73 \%$, hypertension in $40 \%$, impaired glucose tolerance or diabetes mellitus in $60 \%$ and dyslipidemia in $30 \%$ of patients. Insulin levels were increased compared with normal subjects at fasting and after glucose load, suggesting a state of insulin resistance with hyperinsulinemia. Finally, the significant correlation between waist-to-hip ratio, expression of abdominal obesity or fasting insulin levels and most metabolic abnormalities confirmed the role of visceral obesity and insulin resistance in the persistence of metabolic syndrome and their contribution to the persistence of cardiovascular risk in patients with cured CD despite the long-term normalization of cortisol secretion [8]. The direct consequences of the metabolic syndrome characterizing patients with CS are mainly represented by both cardiac and vascular abnormalities.

\section{Vascular Abnormalities}

The main vascular alteration associated with CS is arterial atherosclerosis. Carotid intima-media thickness (IMT), a strong predictor of systemic atherosclerosis, was found to be significantly increased and associated with premature development of carotid atherosclerotic plaques in patients with $\mathrm{CD}$ during the active phase of the disease [7]. In these patients carotid artery IMT decreased after 1 year of disease remission although it remained abnormal compared with the controls, indicating a persistent cardiovascular risk [7]. The improvement in vascular parameters was associated with partial regression of metabolic abnormalities. Waist-to-hip ratio was the best predictor of IMT in active but not in cured disease. The results of this study suggested that the metabolic syndrome associated with endogenous hypercortisolism may play an important role in determining carotid atherosclerosis. It is worth noting that an increased carotid IMT and prevalence of atherosclerotic plaques compared with the general population were also found in a cohort of patients with CD evaluated after long-term disease remission [8]. In these patients, the carotid vascular damage was significantly and independently correlated with the degree of central adiposity and insulin resistance, demonstrating that these factors mainly contribute to the persistence of vascular damage in patients' cure of CD. In a different study, an IMT significantly higher than in a population matched for cardiovascular risk factors, such as smoking habit, body mass index, blood pressure, glucose and lipid levels, was described in multiple vascular districts [9]. CS has also been found to be associated with a functional alteration of the vascular system. Indeed, an endotheliumdependent flow-mediated vasodilation has been reported to be impaired in patients with CS compared with controls [10]. As to markers of endothelial dysfunction and/ or inflammation, there are studies showing a variable increase of endothelin, vascular endothelial growth factor, cell adhesion molecules, interleukin- 8 and osteoprotegerin, which were found to decrease or normalize after successful surgery. However, the clinical relevance of these markers for cardiovascular risk is still unclear [11, $12]$.

Coagulopathy represents another important factor for the development of arterial atherosclerosis in patients with CS. The hypercoagulable state is considered a crucial factor predisposing patients with CS to thromboembolic events, mostly after surgery [13]. Indeed, glucocorticoid excess increases plasma clotting factors, especially factor VIII and the von Willebrand factor complex, and may affect the multimeric structure of this complex causing an overexpression of abnormally high molecular weight multimers, which enhance both the induced and spontaneous platelet aggregation. Patients with CS also present reduced plasma fibrinolytic capacity largely due 
to an increase in plasminogen activator inhibitor type 1 . An adequate anticoagulant prophylaxis can reverse this prothrombotic state and avoid postoperative thromboembolic events [13].

Hyperhomocysteinemia, an important factor associated with a prothrombotic state and with cardiovascular disease, has been described as one of the features of CS [14]. Moreover, increased levels of homocysteine and, more importantly, decreased levels of taurine, have been described in patients with CD [15]. Taurine has increasingly revealed a protective factor for the cardiovascular system, because it seems to counteract the homocysteineinduced cardiovascular damage [15]. This study suggests that taurine deficiency more than homocysteine excess may contribute to the cardiovascular risk of patients with CS. On the other hand, since no abnormalities of homocysteine and taurine metabolism seem to persist after remission from hypercortisolism, hyperhomocysteinemia and hypotaurinemia seem to be reversible metabolic complications that do not contribute to the persistent and progressively worsening cardiovascular risk of patients cured of CS [15].

Hypertension is a common feature of CS, and it represents an important factor for the development of both vascular and cardiac abnormalities in patients with CS $[16,17]$. Hypertension affects $70-80 \%$ of adults and $50 \%$ of children and adolescent patients. The prevalence of hypertension is related neither to the duration of disease nor to gender, while hypertensive patients were significantly older than normotensive patients. The circadian blood pressure rhythm is invariably altered, showing the typical nondipping patterns (lack of physiological nocturnal fall in blood pressure); this pattern is related to greater end-organ damage and increased mortality. Glucocorticoid-dependent hypertension has a multifactorial pathogenesis: inhibition of vasodilatory system (nitric oxide synthase, prostacyclin and kinin-kallikrein), activation of the renin-angiotensin system, increased vascular reactivity to the vasoconstricting stimulation of catecholamines, inhibition of peripheral catabolism of catecholamines, in particular norepinephrine. In addition, the increased cortisol levels may exceed the capacity of the renal enzyme $11 \beta$ hydroxysteroid dehydrogenase type 2 to inactivate cortisol, making it available for binding to the mineralocorticoid receptors, thus behaving like an aldosterone excess. The blood pressure response to antihypertensive therapy is not satisfactory unless normal cortisol levels are restored. In adults, hypertension can persist even after remission due either to a possible coexistence with essential hypertension or to permanent damage incurred by cortisol in the vascular microcirculation $[16$, 17].

\section{Cardiac Abnormalities}

Cardiac function in CS has not been studied much in CS. However, a study evaluating patients with active CS demonstrated the presence of an abnormal left ventricular (LV) geometry with increased LV mass index and relative wall thickness, which was associated with LV diastolic dysfunction, mainly characterized by an impaired relaxation and LV filling pattern, but preserved LV ejection fraction and systolic function [18]. Recently, it has been demonstrated with two-dimensional speckle tracking strain imaging that the abnormalities in the LV structure and function in patients with CS are reversible upon normalization of corticosteroid excess [19]. In this study, a significant reduction in LV mass and relative wall thickness together with a normalization of LV diastolic function was described in patients with CS after disease remission; in particular, there is an early improvement in longitudinal and circumferential shortening and late regression of LV hypertrophy. These changes in myocardial properties are independent of changes in blood pressure, since after normalization of cortisol levels, no significant changes in blood pressure were observed during follow-up. Apparently, restoration of normal cortisol secretion can completely normalize cardiac dysfunction, whereas cardiovascular risk factors, including hypertension or dyslipidemia, persisted in a group of patients despite optimal medical treatment [19]. An alteration of the sympathovagal balance of heart rate variability has also been found to be associated with an increased risk of cardiac events. It is worth noting that patients with CS have been found to display a sympathovagal imbalance characterized by relatively increased parasympathetic activity compared with the controls [20], which suggests that a larger use of Holter electrocardiogram monitoring is warranted in order to reveal the occurrence of silent arrhythmias and/or myocardial ischemia in patients with CS.

\section{Conclusions}

The studies evaluating the vascular and cardiac structure and function in CS suggest that in the clinical management of patients with CS the focus should be on iden- 
Fig. 1. Schematic representation of the relation between glucocorticoid excess and the development of cardiac and vascular abnormalities responsible for the increased cardiovascular risk in patients with CS.

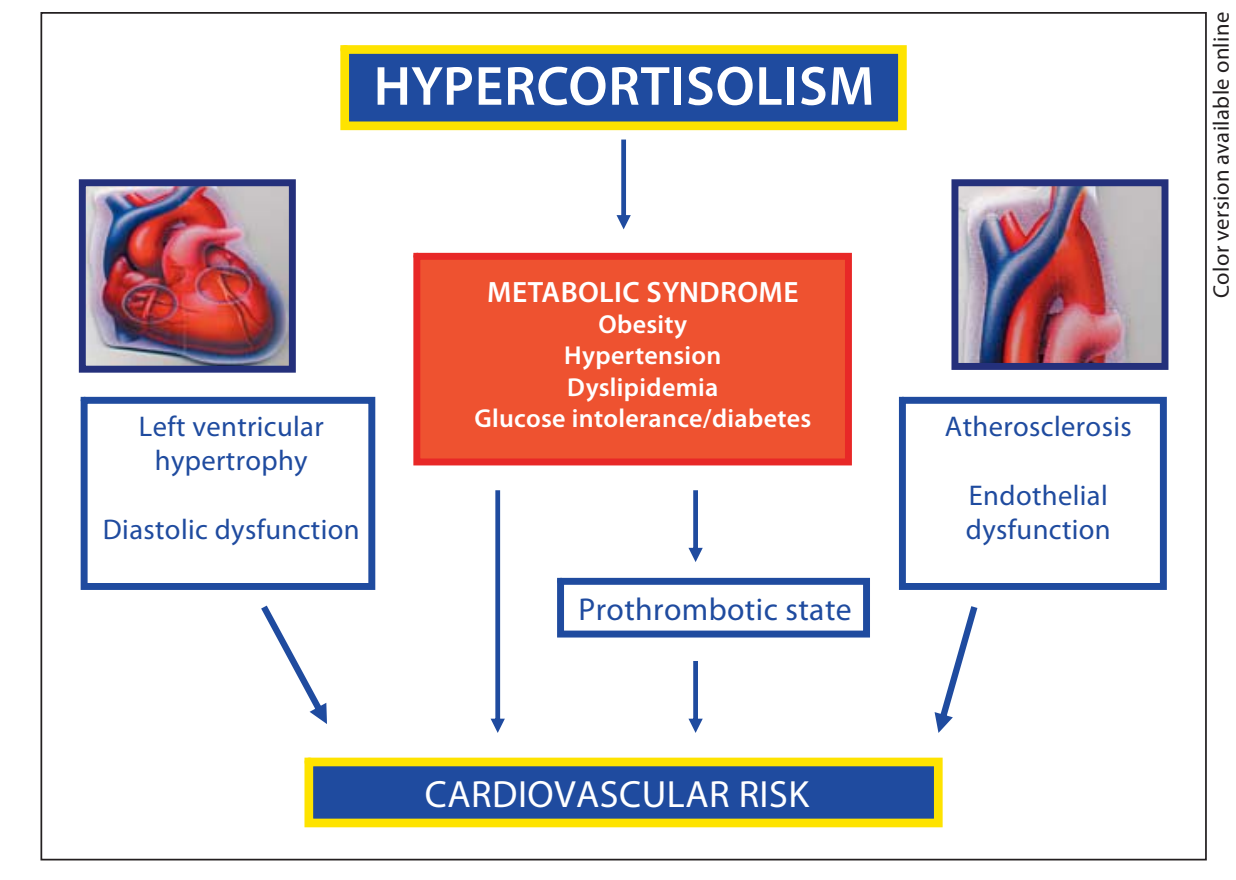

tifying the global cardiovascular risk and the aim should be to control not only hypertension but also other correlated risk factors, such as obesity, glucose intolerance, insulin resistance, dyslipidemia, endothelial dysfunction and the prothrombotic state. Figure 1 shows a schematic representation of the relationship between glucocorticoid excess and cardiovascular risk. Considering that remission from hypercortisolism is often difficult to achieve and that the cardiovascular risk can persist even during disease remission, care and control of all cardiovascular risk factors should be one of the primary goals during the follow-up of these patients.

\section{Disclosure Statement}

The authors have nothing to disclose.

\section{References}

1 Pivonello R, De Martino MC, De Leo M, Lombardi G, Colao A: Cushing's syndrome. Endocrinol Metab Clin North Am 2008;37: 135-149.

2 Exabe J, Vazquez JA: Morbidity and mortality in Cushing's disease: an epidemiological approach. Clin Endocrinol (Oxf) 1994;4: 479-484.

>3 Lindholm J, Juul S, Jorgensen JO, Astrup J, Bjerre P, Feldt-Rasmussen U, Hagen C, Jørgensen J, Kosteljanetz M, Kristensen L, Laurberg P, Schmidt K, Weeke J: Incidence and late prognosis of Cushing's syndrome: a population-based study. J Clin Endocrinol Metab 2001;86:117-123.

4 Pivonello R, De Martino MC, De Leo M, Tauchmanovà L, Faggiano A, Lombardi G, Colao A: Cushing's syndrome: aftermath of the cure. Arq Bras Endocrinol Metab 2007; 51:1381-1391.

5 Pivonello R, Faggiano A, Lombardi G, Colao A: The metabolic syndrome and cardiovascular risk in Cushing's syndrome. Endocrinol Metab Clin North Am 2005;34:327-339.

6 Cameron AJ, Shaw JE, Zimmet PZ: The metabolic syndrome: prevalence in worldwide population. Endocrinol Metab Clin North Am 2004;33:351-375.

7 Faggiano A, Pivonello R, Spiezia S, De Martino MC, Filippella M, Di Somma C, Lombardi G, Colao A: Cardiovascular risk factors and common carotid artery calibre and stiffness in patients with Cushing's disease during active disease and 1 year after disease remission. J Clin Endocrinol Metab 2003;88: 2527-2533.
8 Colao A, Pivonello R, Spiezia S, Faggiano A, Ferone D, Filippella M, Marzullo P, Cerbone G, Siciliani M, Lombardi G: Persistence of increased cardiovascular risk in patients with Cushing's disease after five years of successful cure. J Clin Endocrinol Metab 1999;84:2664-2672.

9 Albiger N, Testa RM, Almoto B, Ferrari M, Bilora F, Petrobelli F, Pagnan A, Mantero F, Scaroni C: Patients with Cushing's syndrome have increased intima-media thickness at different vascular levels: comparison with a population matched for similar cardiovascular risk factors. Horm Metab Res 2006;38:405-410. 
10 Baykan M, Erem C, Gedikli O, Hacihasanoglu A, Erdogan T, Kocak M, Kaplan S, Kiriș A, Orem C, Celik S: Impairment of flow-mediated vasodilatation of brachial artery in patients with Cushing's syndrome 2007;25: 182-190.

$\checkmark 11$ Ermetici F, Malavazos AE, Corbetta S, Eller Vainicher C, Cannavò S, Corsi MM, Ambrosi B: Soluble adhesion molecule levels in patients with Cushing's syndrome before and after cure. J Endocrinol Invest 2008;31:389-392.

$\checkmark 12$ Kristo C, Ueland T, Godang K, Aukrust P, Bollerslev J: Biochemical markers for cardiovascular risk following treatment in endogenous Cushing's syndrome. J Endocrinol Invest 2008;31:400-405.

-13 Boscaro M, Sonino N, Scarda A, Barzon L, Fallo F, Sartori MT, Patrassi GM, Girolami A: Anticoagulant prophylaxis markedly reduces thromboembolic complications in Cushing's syndrome. J Clin Endocrinol Metab 2002;87:3662-3666.
14 Terzolo M, Allasino B, Bosio S, Brusa E, Daffara F, Ventura M, Aroasio E, Sacchetto G Reimondo G, Angeli A, Camaschella C: Hyperhomocysteinemia in patients with Cushing's syndrome. J Clin Endocrinol Metab 2004;89:3745-3751.

15 Faggiano A, Melis D, Alfieri R, De Martino M, Filippella M, Milone F, Lombardi G, Colao A, Pivonello R: Sulfur amino acids in Cushing's disease: insight homocysteine and taurine levels in patients with active and cured disease. J Clin Endocrinol Metab 2005; 90:6616-6622.

16 Magiakou MA, Smyrnaki P, Chrousos GP Hypertension in Cushing's syndrome. Best Pract Res Clin Endocrinol Metab 2006;20: 467-482.
Pecori Giraldi F, Toja PM, De Martin M, Maronati A, Scacchi M, Omboni S, Cavagnini F, Parati G: Circadian blood pressure profile in patients with active Cushing's disease and after long-term cure. Horm Metab Res 2007; 39:908-914.

18 Muiesan ML, Lupia M, Salvetti M, Grigoletto C, Sonino N, Boscaro M, Rosei EA, Mantero F, Fallo F: Left ventricular structural and functional characteristics in Cushing's syndrome. J Am Coll Cardiol 2003;41:22752279.

-19 Pereira AM, Delgado V, Romijn JA, Smit JW, Bax JJ, Feelders RA: Cardiac dysfunction is reserved upon successful treatment of Cushing's syndrome. Eur J Endocrinol 2010;162: 331-340.

20 Fallo F, Maffei P, Dalla Pozza A, Carli M, Della Mea P, Lupia M, Rabbia F, Sonino N: Cardiovascular autonomic function in Cushing's syndrome. J Endocrinol Invest 2009;32:41-45. 